\title{
Minimizing Human Error in Radiopharmaceutical Preparation and Administration via a Bar Code-Enhanced Nuclear Pharmacy Management System
}

\author{
John L. Hakala, Joseph C. Hung, and Elton A. Mosman \\ Division of Nuclear Medicine, Department of Radiology, Mayo Clinic, Rochester, Minnesota
}

\begin{abstract}
The objective of this project was to ensure correct radiopharmaceutical administration through the use of a bar code system that links patient and drug profiles with on-site information management systems. This new combined system would minimize the amount of manual human manipulation, which has proven to be a primary source of error. The most common reason for dosing errors is improper patient identification when a dose is obtained from the nuclear pharmacy or when a dose is administered. A standardized electronic transfer of information from radiopharmaceutical preparation to injection will further reduce the risk of misadministration. Methods: Value stream maps showing the flow of the patient dose information, as well as potential points of human error, were developed. Next, a future-state map was created that included proposed corrections for the most common critical sites of error. Transitioning the current process to the future state will require solutions that address these sites. To optimize the future-state process, a bar code system that links the on-site radiology management system with the nuclear pharmacy management system was proposed. A bar-coded wristband connects the patient directly to the electronic information systems. Results: The bar code-enhanced process linking the patient dose with the electronic information reduces the number of crucial points for human error and provides a framework to ensure that the prepared dose reaches the correct patient. Although the proposed flowchart is designed for a site with an in-house central nuclear pharmacy, much of the framework could be applied by nuclear medicine facilities using unit doses. Conclusion: An electronic connection between information management systems to allow the tracking of a radiopharmaceutical from preparation to administration can be a useful tool in preventing the mistakes that are an unfortunate reality for any facility.
\end{abstract}

Key Words: bar code technology; radiopharmacy; medical event; radiation safety; radiopharmaceuticals

J Nucl Med Technol 2012; 40:183-186

DOI: 10.2967/jnmt.111.097105

$\mathbf{M}$

istakes involving the administration of radiopharmaceuticals are an inherent risk in the practice of nuclear medicine. An administration mistake in nuclear medicine may

Received Aug. 17, 2011; revision accepted Feb. 8, 2012.

For correspondence contact: Joseph C. Hung, Department of Radiology,

Mayo Clinic, 200 First St. SW, Rochester, MN 55905-0001.

E-mail: jhung@mayo.edu

Published online Jun. 5, 2012.

COPYRIGHT (c) 2012 by the Society of Nuclear Medicine and Molecular Imaging, Inc. include, but is not limited to, dispensing a radiopharmaceutical to the wrong patient, giving the incorrect radiopharmaceutical for a given study, or dispensing the incorrect dose of the desired radiopharmaceutical (1). Each of these scenarios is seriously detrimental and should be corrected by any means necessary (2). Because of these potential mistakes, and reports of actual examples of error, an evaluation of sources of human error in nuclear medicine was deemed necessary.

At the root of many incident reports lies a source of error directly related to human interaction. Although humans may always play the critical role in the field of nuclear medicine, corrective action must be taken to secure the points of radiopharmaceutical preparation and administration, where this interaction is most fallible. The purpose of this research was to pinpoint sources of error in the transfer and administration of radiopharmaceuticals, to create solutions that eliminate these potential problems, and to evaluate the solutions' ability to prevent mistakes.

\section{MATERIALS AND METHODS}

\section{Creation of Value Stream Maps}

To understand where and why a weak spot exists in the practice of nuclear medicine, the initial step was to break down the flow of information and radiopharmaceuticals from the time of preparation to the point of administration. Both generalized and sitespecific value stream maps were created.

Developed as part of the lean Six Sigma quality control methodology (Motorola Solutions, Inc.), a value stream map is a useful tool for analyzing the flow and transfer of information and items. This tool was determined to be the best course of action for this project, because within a nuclear medicine facility, correct information and radiopharmaceutical transfer is critical.

In discerning where the actual weak spots exist, and in discussing solutions to these sites of error, we needed to obtain many perspectives from different operations to avoid bias or impartial results. The participants included a board-certified nuclear pharmacist, a nuclear medicine supervisor, a lead nuclear pharmacy technologist, and a certified nuclear medicine technologist.

To build a value stream map, participants carefully observed and studied the flow of radiopharmaceutical and information transfer from the time a dose was prepared to the time it was administered. Each step within the process was given an individual box on the map, and these boxes were arranged in chronologic order.

Each step of the value stream map was thoroughly discussed, and from it several sources of human error were identified. These 
points were highlighted and used as a map to guide the implementation of the proposed solutions.

\section{Implementation of Bar Code Technology}

On site, 2 types on management systems are used. One is a nuclear pharmacy management system, Optility (Synergic Solutions, Inc.), which technologists use primarily for creating radiopharmaceutical kits, preparing patient doses, and monitoring the status of patient doses as filled or unfilled. The other management system used, RIMS (Radiology Information Management System), was developed by our institution and specifically designed for the operation of our radiology department. It keeps records of completed and upcoming patient scans, including the type of examination, the staff involved with the study, and the radiopharmaceutical dose.

Both management systems already had bar code capabilities. Our strategy was then to link the 2 systems so that information from the patient's study would be electronically connected with the records and inventory systems. As an example of a particularly useful parameter, each patient was assigned an identifying number that was specific to the patient and to the procedure the patient was to receive. This number was printed in bar code format and was readable by both management systems. To eliminate what was found to be the primary source of mistakes (i.e., verbal verification), this bar code was printed on a patient-specific wristband on arrival and was scanned just before administration as a final electronic verification, along with verbal verification.

\section{Verification of Dispensed and Administered Radiopharmaceutical}

Another critical point to be addressed was the passing of the radiopharmaceutical from the nuclear pharmacy quality control room to the administering technologist. The current method was visual verification of patient name on identical patient information pages. The solution was to place a bar-coded sticker with the patient identifier number on the radiopharmaceutical syringe shield.

\section{Dose Verification}

From the evaluation of the general value stream map, a third solution was devised that would eliminate errors caused by manual entry of dose information. Many nuclear medicine laboratories do not use dose calibrators that are integrated with nuclear pharmacy software. Accidental manual error occurs by a simple typing mistake or an improper selection of data. The solution to manual-entry errors was to fully integrate the nuclear pharmacy management software with the dose calibrator, ensuring that the correct dose was being entered by the technologist.

\section{RESULTS}

\section{Value Stream Maps}

To pinpoint the most common sites of human error, the primary objective was to create a stepwise map of how a radiopharmaceutical is transferred from the time it is prepared until the time it is administered to the patient (Fig. 1). Because our institution contains several in-house nuclear pharmacy laboratories for preparing and dispensing various radiopharmaceuticals, radiopharmaceutical transfer could be evaluated from the point of synthesis of multipleuse radiopharmaceutical kit preparations.

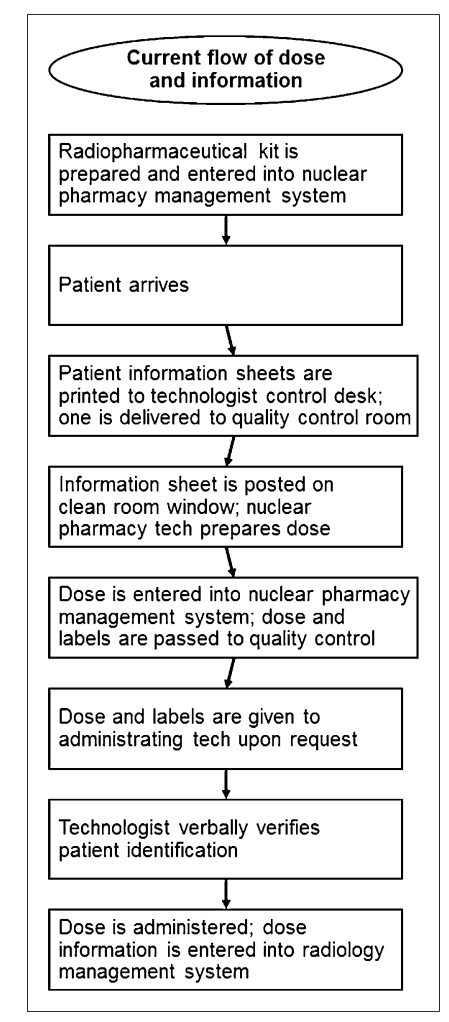

FIGURE 1. Value stream map of typical flow of radiopharmaceuticals from preparation to administration.

Human error was most common when either verbal or visual verification was required to pass along or dispense the radiopharmaceutical (Fig. 2) or when a technologist had to manually enter dose information into the nuclear pharmacy management systems.

The major corrective solutions were charted alongside the initial value stream map (Fig. 3). From that stage, a futurestate map was created that documented how the ideal and error-minimized flow of radiopharmaceuticals and patient information would occur (Fig. 4). The future-state map served not only as a guide for technologists not familiar with the new process but as an analysis tool for the implemented solutions.

The unanimous decision on addressing the corrective actions was to implement a strategy in which bar code technology is used in a series of electronic checkpoints that follows the patient and dose from check-in to radiopharmaceutical administration.

\section{Bar Code Technology}

The new bar code-enhanced process linking the patient dose with the electronic information reduces the number of crucial points for human error and provides a framework to ensure the prepared dose reaches the correct patient. Specific connections were made that allowed for easily implemented solutions, with tangible effects on the safety and accuracy of nuclear medicine administrations.

\section{Verification of Dispensed and Administered Radiopharmaceutical}

When the administering technologist receives the radiopharmaceutical, the dose bar code is scanned, as well as the 


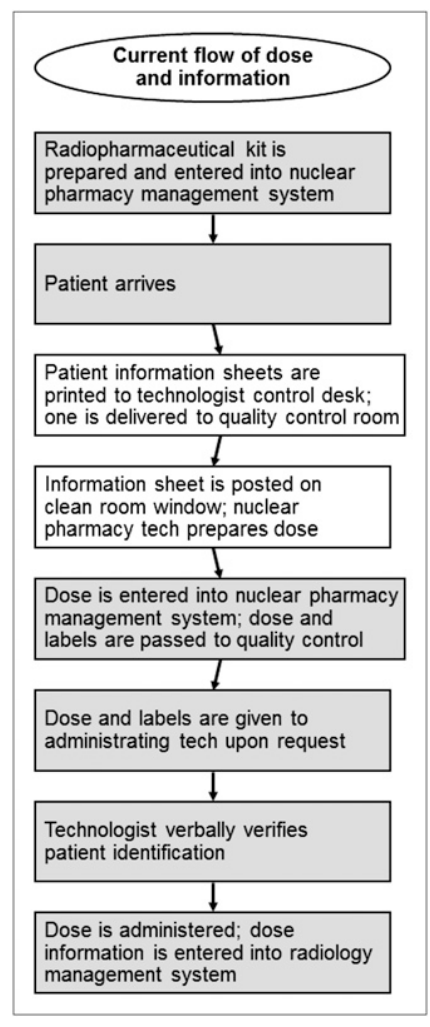

FIGURE 2. Points of human error, highlighted, in value stream map of radiopharmaceutical transfer.

patient information page bar code. An unsuccessful match will not allow the technologist to dispense the dose.

\section{Dose Verification}

Direct integration of the dose calibrator with the management system will also ensure that the correct isotope is selected on the dose calibrator, as this is a feature on current nuclear pharmacy management software. This step is viable only if proper maintenance and quality control measures are taken for the dose calibrator.

\section{DISCUSSION}

Avoiding mistakes related to radiopharmaceutical administration is of critical importance in a nuclear medicine department. The worst consequence of improper preparation and administration is a medical event involving radiopharmaceuticals (3). There have been well-documented cases of improper administration of therapeutic doses of ${ }^{131} \mathrm{I}$, resulting in seriously detrimental effects on the patient's physiology (4). These types of events are reported to the Nuclear Regulatory Commission and made publicly available. Such reports can lead to a risk of depreciated reputation and trust in a facility.

Immediate quantitative data are not available, but the number of mistakes made in a nuclear medicine department is expected to decrease significantly if the discussed solutions are implemented. Although mistakes may be prevented by detail-oriented staff, further statistical analysis may be necessary to determine the long-term success of an electronic bar-coded system.

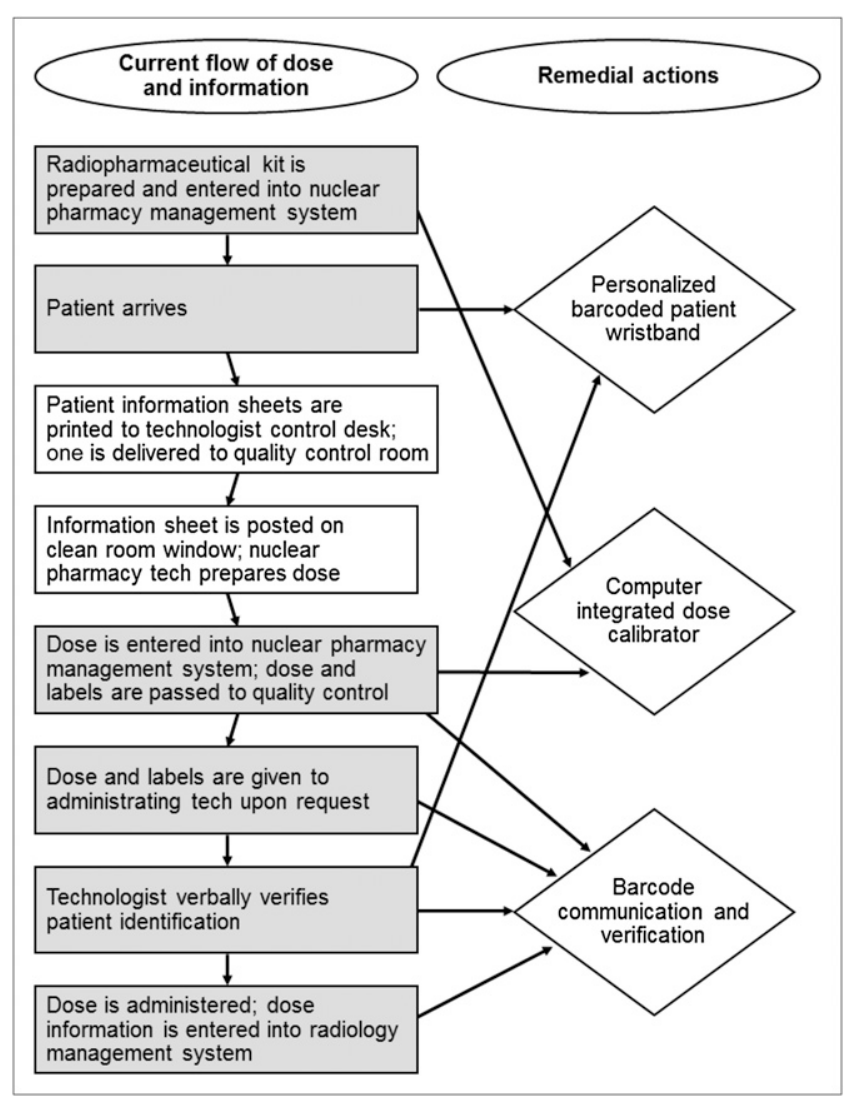

FIGURE 3. Proposed corrections to points of human error, as related to value stream map of radiopharmaceutical transfer.

The proposed process flow was designed for a site with an in-house central nuclear pharmacy laboratory; however, much of the framework could be applied by nuclear medicine facilities using unit doses. The solutions are generally costeffective, as no overhauls of data transfer devices or software programming strategies are involved. Short-term additions include long-range wireless bar code scanners and necessary hardware for assembly. Sites requiring computer connection to dose calibrators may need to evaluate the systems for compatibility and the essential data transfer hardware.

Bar code technology makes many of these solutions viable for many nuclear medicine sites. A checkpoint system may be as simple as scanning identical bar codes into spreadsheet software. Current bar code scanners are able to read many types of bar codes, and encoding of data into bar codes is a relatively user-friendly task (5).

With technologic changes to a system, there are often technical challenges to overcome. In this project, there were both hardware and software issues to be resolved. When a dose calibrator is being integrated with a nuclear pharmacy management system, both hardware and software technical issues arise. Not all dose calibrators can connect with a personal computer, but those that do often require an RS-232-to-universal serial bus connection. Computer location, cable length, and computer connection port availability are all considerations. The major software consideration also involves the computer-integration solution. Having the hard- 


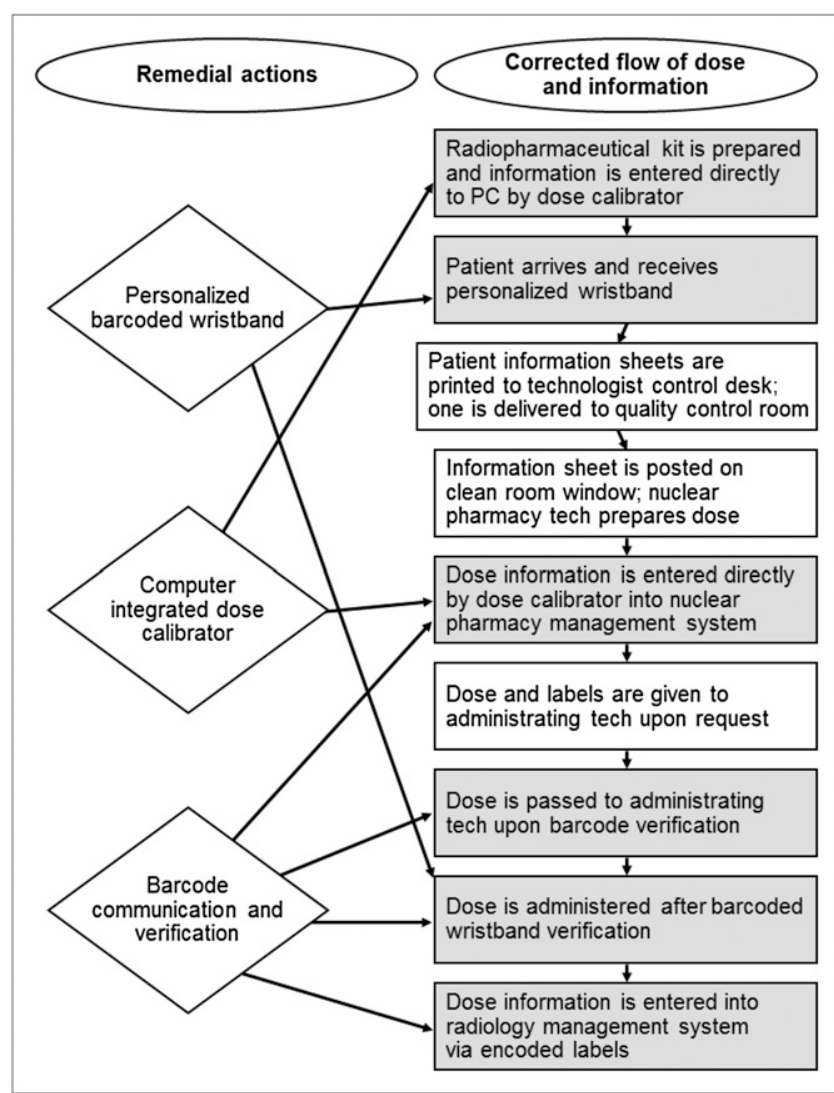

FIGURE 4. Future-state map updated by corrective actions to points of human error.

ware and connection alone does not integrate the dose calibrator to the computer. Software interfacing between the 2 devices must be written either by the proprietary software programmers or by highly trained technologists. Although many software programs have this feature, our version of Optility did not. This deficiency may prompt either a software upgrade, which was deemed possible by technical support from Optility, or a change in nuclear pharmacy management systems.

The climate of electronic interfaces is rapidly changing. As more electronics rapidly become wireless and network capabilities improve, there is room for advancement in nuclear medicine departments. Open wireless technology, instant data feeds, and improved communications may all play a role in the transfer and administration of radiopharmaceuticals and help to improve the safety of related processes.

\section{CONCLUSION}

The creation of an electronic connection between management information systems to allow the tracking of a radiopharmaceutical from time of preparation to administration can be a useful tool in preventing the mistakes that are an unfortunate reality for any nuclear medicine facility. Although efforts to electronically track patient doses and information may alleviate many of the critical error points, changes will need to be continually made in order to keep up with the evolution of clinical practice in the dynamic field of nuclear medicine. The safety and accuracy of patient studies should always be the foremost concern of any nuclear medicine department, and appropriate measures need to be taken to reduce, and maybe someday eliminate, any possible source of administration mistakes.

\section{ACKNOWLEDGMENTS}

We thank Thomas J. Herold, CNMT, and William M. Oswald, CNMT, for their assistance with research in the nuclear pharmacy laboratory and the general nuclear medicine laboratory, respectively, in the Division of Nuclear Medicine-Department of Radiology, Mayo Clinic, Rochester, Minnesota. This paper was presented at the 58th annual meeting of the Society of Nuclear Medicine, San Antonio, Texas, on June 6, 2011. No other potential conflict of interest relevant to this article was reported.

\section{REFERENCES}

1. Diagnostic nuclear medicine: misadministrations. International Atomic Energy Agency Web site. Available at: http://rpop.iaea.org/RPOP/RPoP/XPages/XPages2/ Content/InformationFor/HealthProfessionals/3_NuclearMedicine/Diagnostic_nuclear_ medicine_-_accidents.htm. Accessed May 7, 2012.

2. Society of Nuclear Medicine procedure guideline for the use of radiopharmaceuticals. Society of Nuclear Medicine Web site. Available at: http://interactive.snm. org/docs/pg_ch14_0403.pdf. Accessed May 7, 2012.

3. Risks associated with medical events. United States Nuclear Regulatory Commission Web site. Available at: http://www.nrc.gov/reading-rm/doc-collections/factsheets/risks-assoc-medical-events.html. Published April 2006. Updated March 15, 2011; Accessed May 7, 2012.

4. Chow SM. Side effects of high-dose radioactive iodine for ablation or treatment of differentiated thyroid carcinoma. J HK Coll Radiol Web site. Available at: http:// www.hkcr.org/publ/Journal/vol8no3/full/127-135\%20Side.pdf. Published August 2005. Accessed May 7, 2012.

5. Cummings J, Ratko T, Matuszewski K. Barcoding to enhance patient safety. Patient Safety and Quality Healthcare Web site. Available at: http://www.psqh. com/sepoct05/barcodingrfid1.html. Published September/October 2005. Accessed May 7, 2012 